\title{
Experimental study on bone marrow mesenchymal stem cells for the treatment of acute pancreatitis in
}

\section{rats}

\begin{abstract}
Explored the appropriate way for bone marrow mesenchymal stem cells (bmMSCs) infuse in rat models of severe acute pancreatitis. Severe AP was induced in SprageDawley rats by the sodium taurocholate bile duct retrograde injection. Rats were randomly divided into five groups: control group, SAP group, SAP+bmMSCs (tail vein) group, SAP+bmMSCs (intraperitoneal) group and SAP+bmMSCs (tail vein + intraperitoneal) group. In bmMSCs infused groups, bmMSCs labeled with DAPI were injected via the tail vein, intraperitoneal and via tail vein + intraperitoneal respectively $3 \mathrm{~h}$ after SAP. Rats were sacrificed on days 1, 2 and 3, and pancreatic tissues and the blood were collected. The levels of serum anti-inflammatory cytokines IL-10 and inflammatory cytokines (IL-1 $\beta$, TNF- $\alpha$, IL-6) were determined. Pathological changes of the pancreas (HE staining) were observed under light microscope. Positioning of DAPI labeled bmMSCs In vivo were detected under fluorescence microscope at the same time. The pancreas in SAP group showed significantly massive hemorrhage, edema, inflammation, and necrosis compared with control group. The inflammation, edema, hemorrhage and necrosis in each model of pancreatitis were reduced significantly in $\mathrm{SAP}+$ bmMSCs group as compared to SAP group $(\mathrm{P}<0.05)$. In bmMSCs transplanted group, bmMSCs remarkably reduced the levels of pro-inflammatory cytokines (TNF- $\alpha$, IL-6, and IL-1 $\beta$ ), on the contrary increased the levels of anti-inflammatory cytokines (IL-10) in SAP rats. Infused bmMSCs through tail vein + intraperitoneal indicate a better recovery than through tail vein or intraperitoneal alone. Compared with intraperitoneal injection method, more bmMSCs were observed to be presented in pancreas when bmMSCs were injected through tail vein + intraperitoneal or tail vein injection. In a rat model of SAP, transplantation of bm-MSCs through tail vein + intraperitoneal significantly inhibits inflammation and decreases pancreatic injury secondary to SAP. It was a better method for pancreatitis.
\end{abstract}

Volume 2 Issue I - 2018

\author{
Hanguang Dong,' Xueying Tan,' Mingjie Fu, ${ }^{2}$ \\ Jie Zhao, ${ }^{3}$ Qian Ge, 'Yigang Chen, ${ }^{4}$ Ying $\mathrm{Li}^{\prime}$ \\ 'Department of Hepatobiliary Surgery, China \\ ${ }^{2}$ Surgical Intensive Care Unit, First Affiliated Hospital of Zhejiang \\ University, China \\ ${ }^{3}$ Department of Anesthesiology, China \\ ${ }^{4}$ Department of General Surgery, The Affiliated Wuxi No.2 \\ People's Hospital of Nanjing Medical University, China \\ *\#both authors are equal contributions
}

\begin{abstract}
Correspondence: Yigang Chen, Department of General Surgery, The Affiliated Wuxi No.2 People's Hospital of Nanjing Medical University, China, Tel +86-5 10-6668I222, Fax +86-5 I06668I222, Email chyg7676@alumni.sjtu.edu.cn

Ying Li, Department of Ophthalmology, Qingdao Municipal Hospital, No. I Jiaozhou Road, Qingdao 200240 PR, China, Tel +86-5 I0-6668I 222, Fax+86-532-82789I59, Email13506187399@।39.com
\end{abstract}

Received: December 28, 2017 | Published: January 05, 2018

Keywords: acute pancreatitis, bone marrow mesenchymal stem cells, endothelin, nitric oxide

Abbreviations: AP, acute pancreatitis; SAP, severe acute pancreatitis; TNF, tumor necrosis factor; $\mathrm{NO}$, nitric oxide; SD, sprague dawley

\section{Introduction}

Acute pancreatitis (AP) is a rapid onset and disease progression, and approximately $15-20 \%$ of patients with AP may develop severe acute pancreatitis (SAP) which has the high mortality rate. ${ }^{1}$ It is now generally accepted that the pathogenesis of AP start from the activation of pancreatin in pancreatic acinar cells and then induces both local and systemic inflammation. ${ }^{2}$ Tumor necrosis factor (TNF), C-reactive protein, interleukin (IL)-1, IL-8, IL-6, endothelin, nitric oxide (NO) and other inflammatory factors are thought to be involved in both the genesis and progression of AP. Inflammatory factors play a crucial role in the progression from slight acute pancreatitis to severe acute pancreatitis. Although efforts began to implement the appropriate method for the treatment of acute pancreatitis, mortality rate of SAP has not substantially decreased during the past two decades. ${ }^{3}$ For this reason, adult stem cells especially mesenchymal stromal cells (MSCs) have been used in the experimental studies in the treatment of SAP. MSCs are multipotent adult stem cells that can be obtained from many different tissues shown to low or no immunogenicity. MSCs can differentiated into many cells types by providing the suitable microenvironment similar to In vivo., ${ }^{4}$ Plenty of studies confirmed that MSCs are able to ameliorate pancreatic injury, inhibit systemic inflammation and promote tissue repair in $\mathrm{AP}^{6,7}$ Other studies revealed that MSCs could relieve pancreatitis-associated kidney damage ${ }^{8,9}$ or pancreatitis-associated lung injury. ${ }^{10,11}$ Unfortunately, these studies did not highlight the appropriate way for the stem cells infuse In vivo. To refine these approaches, we transplanted bmMSCs through tail vein, intraperitoneal and tail vein combined with intraperitoneal respectly and then position of bmMSCs, compared pancrearic injury and inflammatory response.

\section{Materials and methods}

\section{Animals}

Healthy Sprague Dawley (SD) rats weighing 200-250g were provided by Shandong Lukang by the Experimental Animal Room of pharmaceutical companies, animal certificate (SLXK Lu 20080002), the experiment disposal of animals found in the animal ethics standards. All the animals were fed under 12-h light-dark cycle conditions with temperature maintained at $20 \pm 2^{\circ} \mathrm{C}$. All experiments protocols were approved by the Animal Ethical Committee of institute. 


\section{Materials and reagents}

Fetal bovine serum (Gibco Company), Dulbecco's modified Eagle's medium (DMEM; Hyclone Corporation, United States), 4,6-diamidino-2-phenylindole (DAPI; Roche, Switzerland), IL10 ELISA for rats, and TNF- $\alpha$ ELISA kit for rats (all ELISAs from GBD company, USA). $0.25 \%$ trypsin $+0.02 \%$ EDTA (Wuhan Boster Company); rabbit anti-rat CD45, CD90 antibody (biolegend company); Histopaque-1077 separation medium (Sigma); flow cytometry (Beckman Coulter Inc.; Type: Epics XL・MCL).

\section{Culture and identification of mesenchymal stem cells}

About $140 \mathrm{~g}$ SD rats were sacrificed and the primary bmMSCs were collected from the femurs and tibias. Following flushing the bone marrow cavity with DMEM-LG medium and plated the cells in basal medium supplemented with $20 \%$ fetal bovine serum, and penicillin $(100 \mathrm{U} / \mathrm{ml}) /$ streptomycin $(100 \mathrm{U} / \mathrm{ml})$ in culture flasks at $37^{\circ} \mathrm{C}$ in $5 \% \mathrm{CO} 2,95 \%$ air in a humidified incubator. When cells confluence reached $>80 \%$ they were harvested by multiple digestions and passages. The surface markers of bmMSCs (CD45 and CD90) were detected by flow cytometry.

\section{Preparation of rat SAP model}

Before surgery, rats were fasted for $12 \mathrm{~h}$ but given water. SAP group: SD rats, weighing 200-250g, were selected for the experiment. A midline incision was made at the abdomen ( $2 \mathrm{~cm}$ in length) and the duodenum was taken out of the abdominal cavity. The pancreas was identified and the duodenal papilla, common bile duct and pancreatic duct were located. Clamp the common bile duct by small clamp, and the shared outlet of common bile duct and pancreatic duct on the duodenum was clamped by bending clamp. Then, $3 \%$ sodium taurocholate $(1 \mathrm{ml} / \mathrm{kg})$ was slowly injected at a speed of $5 \mathrm{~min} / \mathrm{ml}$.

\section{Cell transplantation}

Rats ( $\mathrm{n}=90)$ were randomly divided into control group $(\mathrm{n}=18)$, SAP group $(n=18), S A P+b m M S C s$ (tail vein) group $(n=18)$, SAP+bmMSCs (intraperitoneal) group $(\mathrm{n}=18)$ and SAP+bmMSCs (tail vein + intraperitoneal) group $(n=18)$. In the $\mathrm{SAP}+\mathrm{bmMSCs}$ (tail vein) group and $\mathrm{SAP}+\mathrm{bmMSCs}$ (intraperitoneal)) group, $1 \mathrm{~mL}$ of the bmMSCs suspension (containing approximately $1 \times 10^{6}$ cells labeled with DAPI) were injected into the tail vein or intraperitoneal respectively. In the SAP + bmMSCs (tail vein + intraperitoneal) group, $0.5 \mathrm{~mL}$ of the bmMSCs suspension was injected into the caudal vein and at the same time $0.5 \mathrm{~mL}$ of the bmMSCs suspension was injected into intraperitoneal.

\section{Cell marking}

Third generation bmMSCs were used for subsequent experimentation. bmMSCs were labeled with DAPI(final concentration $50 \mathrm{~g} / \mathrm{l}$ ) and incubated at $37^{\circ} \mathrm{C}$ for $12 \mathrm{~h}$ and observed under fluorescence microscope. Then repeated washing the cells with PBS to remove DAPI, the cells were collected after one more centrifugation. DMEM-F12 medium $(1 \mathrm{ml})$ supplemented containing $1 \times 10^{6}$ MSCs were injected into the rats with SAP through the caudal vein at $3 \mathrm{~h}$.

\section{Morphological examination of the pancreas}

Detection of serum pro-inflammatory cytokines and antiinflammatory cytokines Six rats were collected from all groups at $24 \mathrm{~h}, 48 \mathrm{~h}$ and $72 \mathrm{~h}$ after injection bmMSCs, and pathological scoring was done according to the pathological criteria for pancreatitis (Table 1). Blood samples were collected from the apex of the hearts. The concentrations of serum IL-10, IL-1, IL-6 and TNF- $\alpha$ were determined using ELISA. The pancreatic tissues were flash frozen, and the number of DAPI positive cells were observed under fluorescence microscopy. The body of pancreas was obtained at the indicated time, fixed in $10 \%$ formalin containing phosphate-buffered saline, and embedded in paraffin. Sectioned at $4 \mathrm{~mm}$ thickness sections from each group were analyzed. Samples were stained with H\&E.

\section{Statistical analysis}

All data were expressed as means \pm SEM. Data from experimental groups were compared by Student's t test or analysis of variance. A value of $\mathrm{p}<0.05$ was considered statistically significant and all analyses were performed using Graphpad Prism 5 and SPSS22.0.

Table I Criteria for pathological scoring of the pancreas

\begin{tabular}{|c|c|c|c|c|c|}
\hline \multirow{2}{*}{$\begin{array}{l}\text { Pathological } \\
\text { Findings }\end{array}$} & \multicolumn{5}{|l|}{ Scoring } \\
\hline & 0 & $\mathbf{I}$ & 2 & 3 & 4 \\
\hline 4Edema & No & $\begin{array}{l}\text { Diffused expansion of } \\
\text { lobular septa }\end{array}$ & $\begin{array}{l}\text { Diffused expansion of } \\
\text { lobular septa }\end{array}$ & $\begin{array}{l}\text { Diffused expansion of } \\
\text { acinar septa }\end{array}$ & $\begin{array}{l}\text { Diffused expansion of } \\
\text { cell gap }\end{array}$ \\
\hline $\begin{array}{l}\text { Infiltration of } \\
\text { inflammatory } \\
\text { cells }\end{array}$ & $\begin{array}{l}0-1 \text { white } \\
\text { blood cells/ } \\
\text { HPF }\end{array}$ & $\begin{array}{l}\text { 2-I0 white blood cells/ } \\
\text { HPF }\end{array}$ & $\begin{array}{l}\text { I I-20 white blood } \\
\text { cells/HPF }\end{array}$ & $\begin{array}{l}21-30 \text { white blood } \\
\text { cells/HPF }\end{array}$ & $\begin{array}{l}\geq 3 \text { I white blood cells/ } \\
\text { HPF }\end{array}$ \\
\hline Bleeding & No & $\begin{array}{l}\text { Pancreatic parenchy- } \\
\text { mal bleeding at I-2 } \\
\text { sites }\end{array}$ & $\begin{array}{l}\text { Pancreatic parenchy- } \\
\text { mal bleeding at 3-4 } \\
\text { sites }\end{array}$ & $\begin{array}{l}\text { Pancreatic-parenchy- } \\
\text { mal bleeding at 5-6 } \\
\text { sites }\end{array}$ & $\begin{array}{l}\text { Pancreatic parenchy- mal } \\
\text { bleeding at } \geq 7 \text { sites }\end{array}$ \\
\hline Necrosis & No & I-4 necrotic cells/HP & 5-10 necrotic cells/HP & II-I6 necrotic cells/HP & $\geq 17$ necrotic cells/HP \\
\hline
\end{tabular}




\section{Results}

\section{General morphology of mesenchymal stem cells and the expression of surface markers}

Isolated the cells from bone marrow distributed sparsely on the culture flasks. During the early days of incubation the cells were spindle-shaped morphology. Within 3-5days, the cells displayed small colonies that called colony forming units (Figure 1A). Primary cells reached monolayer confluence and appeared mostly fibroblast like within 10-14 days (Figure 1B). In the later passages, bmMSCs exhibited large and fibroblast-like morphology (Figure 1C). Identification of bmMSCs was by Flow cytometry. The cells analysis data showed that bmMSCs expressed CD54, CD90 but not CD45, the positive rate of CD90 was $99.6 \%$. In contrast, CD45 were negative $(0.56 \%$ and $0.89 \%$, respectively), demonstrating that the purity of the MSCs was $>95 \%$ (Figure 1D).

\section{Histologic Analysis of Pancreas after hcMSCs Infusion}

At $24 \mathrm{~h}, 48 \mathrm{~h}$ and $72 \mathrm{~h}$ points, edema, infiltration of inflammatory cells and necrosis were not observed in the pancreatic tissue in control group. Pancreatic tissue from the SAP group induced by sodium taurocholate showed significant edema and inflammation compared with the control $(P<0.05)$. In bmMSCs infused group the edema formation and inflammatory cell infiltration were significantly reduced, and the improvement of pancreatic in SAP+bmMSCs (tail vein + intraperitoneal) group was better than in SAP+bmMSCs (tail vein) group and SAP+bmMSCs (intrapertoneal) (Figure 2). On the basis of pancreatic parenchymal necrosis, pathological scoring was performed (Figure 3). As showed in Figure 3 the pathological scores
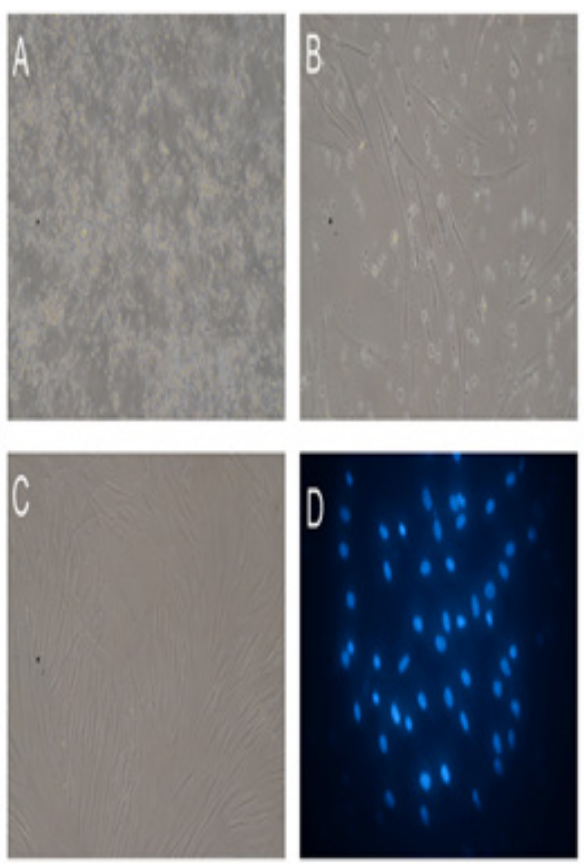

of pancreatic edema, necrosis and infiltration of inflammatory cells in SAP group were markedly higher than those in control group $(\mathrm{P}<0.05)$. In SAP+bmMSCs group, the pancreatic scores were reduced when compared with SAP group. In addition, in SAP+bmMSCs (tail vein + intraperitoneal) group, the pancreatic scores were reduced more significantly than in SAP+bmMSCs (tail vein) and in SAP+bmMSCs (intraperitoneal) group.

\section{Regulation of bmMSCs on inflammatory factors}

In sodium taurocholate -induced SAP models, levels of IL-1 $\beta$, TNF- $\alpha$, IL- 6 and IL-10 in serum were detected by ELISA. Compared with control group, serum IL-1 $\beta$, TNF- $\alpha$ and IL- 6 were obvious increased in SAP group and tended to decrease after injection of bmMSCs treatment. On the contrary, bmMSC increased the level of the anti-inflammatory cytokines such as IL-10 in rats with SAP. Interestingly, bmMSC could better regulate inflammatory mediators and cytokines in rats receiving bmMSCs through tail vein + intraperitoneal compared with receiving bmMSC through tail vein or intraperitoneal alone.

\section{Detection of MSCs in rat pancreas}

DAPI was selected as the labeling system for bmMSCs tracking In vivo. We labeled bmMSCs with DAPI. We further investigated whether bmMSCs migrate into the injured pancreatic after bmMSC transplant by using fluorescence microscopy. DAPI-labeled cells in the bmMSC infused groups displayed blue fluorescence in both SAP + bmMSCs (tail vein + intraperitoneal) group and SAP + bmMSCs (tail vein) group. However there was no cell-specific blue fluorescence was observed in sections from rats that injected DAPI-labeled bmMSC through intraperitoneal.
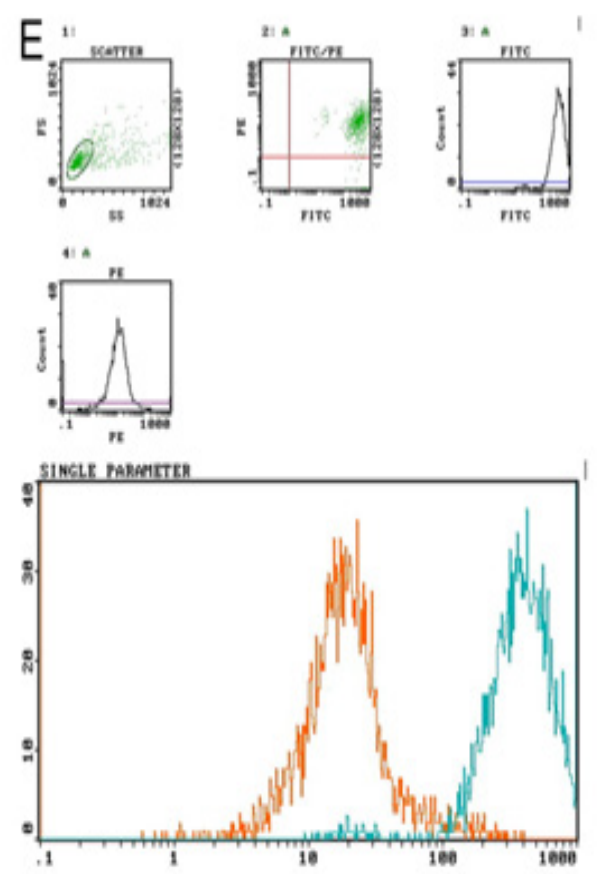

Figure I Isolated cells from bone marrow of rat distributed sparsely on the culture flasks. (A) During the early days of incubation the cells displayed mostly spindle shaped morphology. (B) The small colonies reached monolayer confluence within 10-14 days. (C) Most of these MSCs exhibited large and displayed mostly fibroblast-like morphology in the later passages. (D) labeled MSCs with DAPI. (E) Surface markers of MSCs CD90 was $99.6 \%$. In contrast, CD45 were negative $(0.56 \%$ and $0.89 \%$, respectively) 


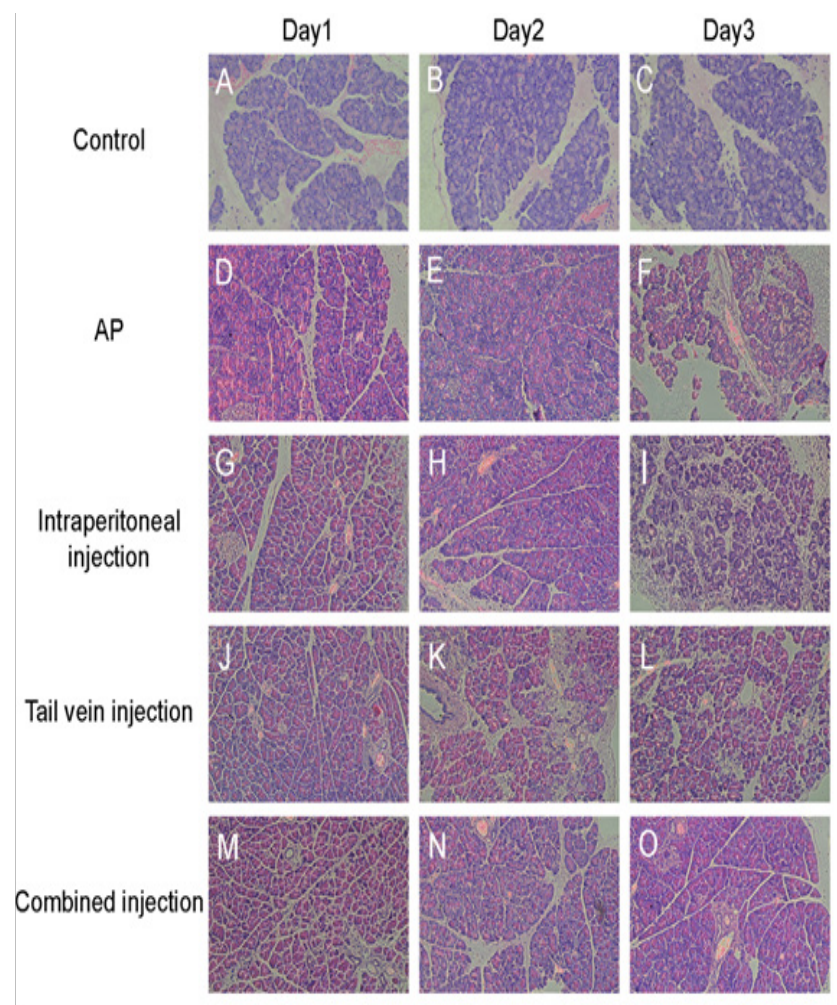

Figure 2 Histological evaluation of pancreas in sodium taurocholate-induced SAP when treated with bmMSCs In vivo $(\times 200)$. A-C, Pancreatic pathology on days I, 2 and 3 in control group; D-F, SAP group on days I, 2 and 3; G-I, SAP + BMSCs (intraperitoneal) group J-L, SAP + bmMSCs (tail vein) group; M-O, SAP + bmMSCs (tail vein + intraperitoneal) group
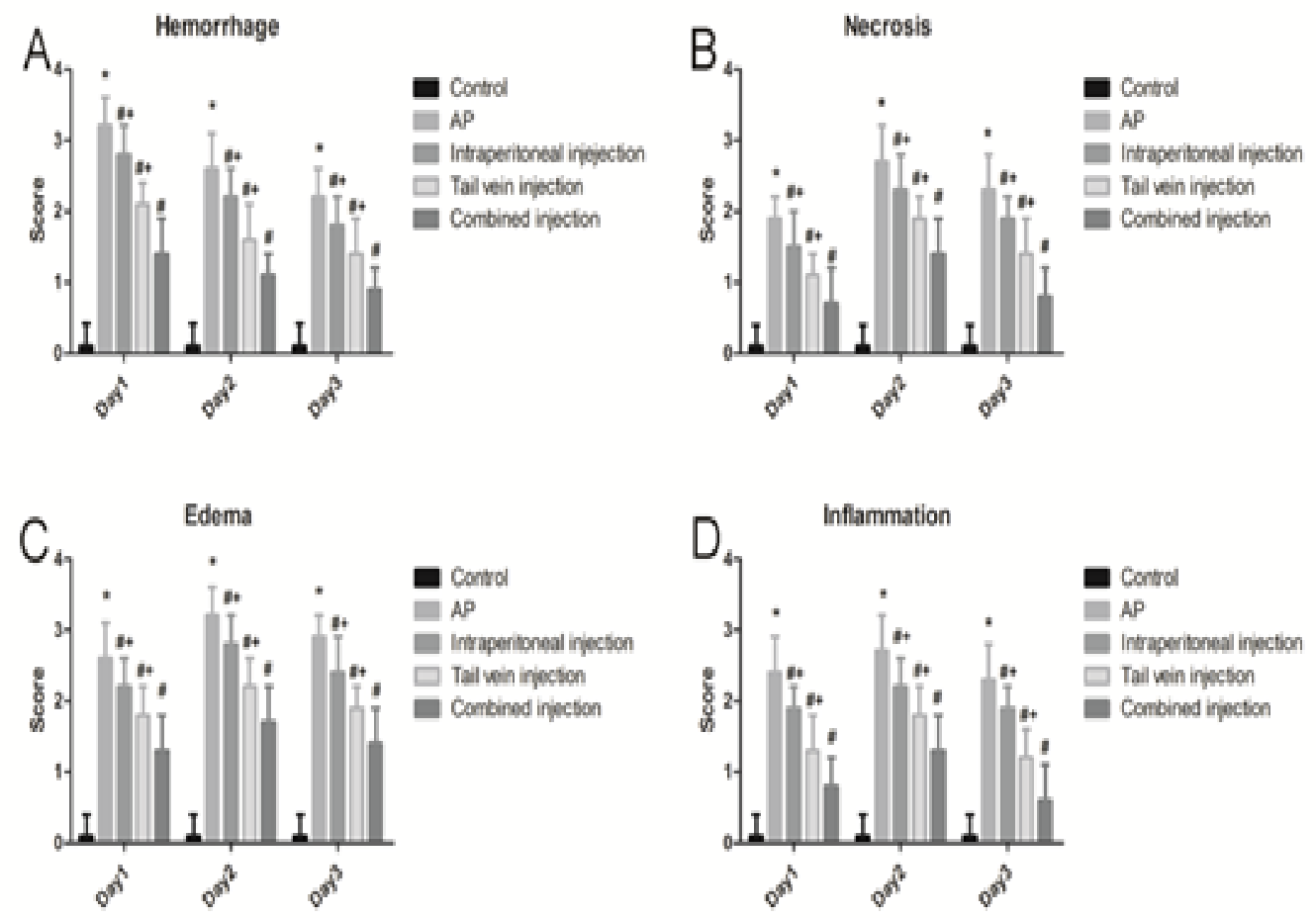

Figure 3 Scoring of pancreatic pathology. A, Score of pancreatic hemorrhage; B, Score of pancreatic necrosis; C, Score of pancreatic edema; D: Score of pancreatic inflammatory cell infiltration. * means $\mathrm{P}<0.05$ when compared with Control group, \# $\mathrm{P}$ means $\mathrm{P}<0.05$ when compared with SAP group. + means $\mathrm{P}<0.05$ when compared with SAP + combined injection group 

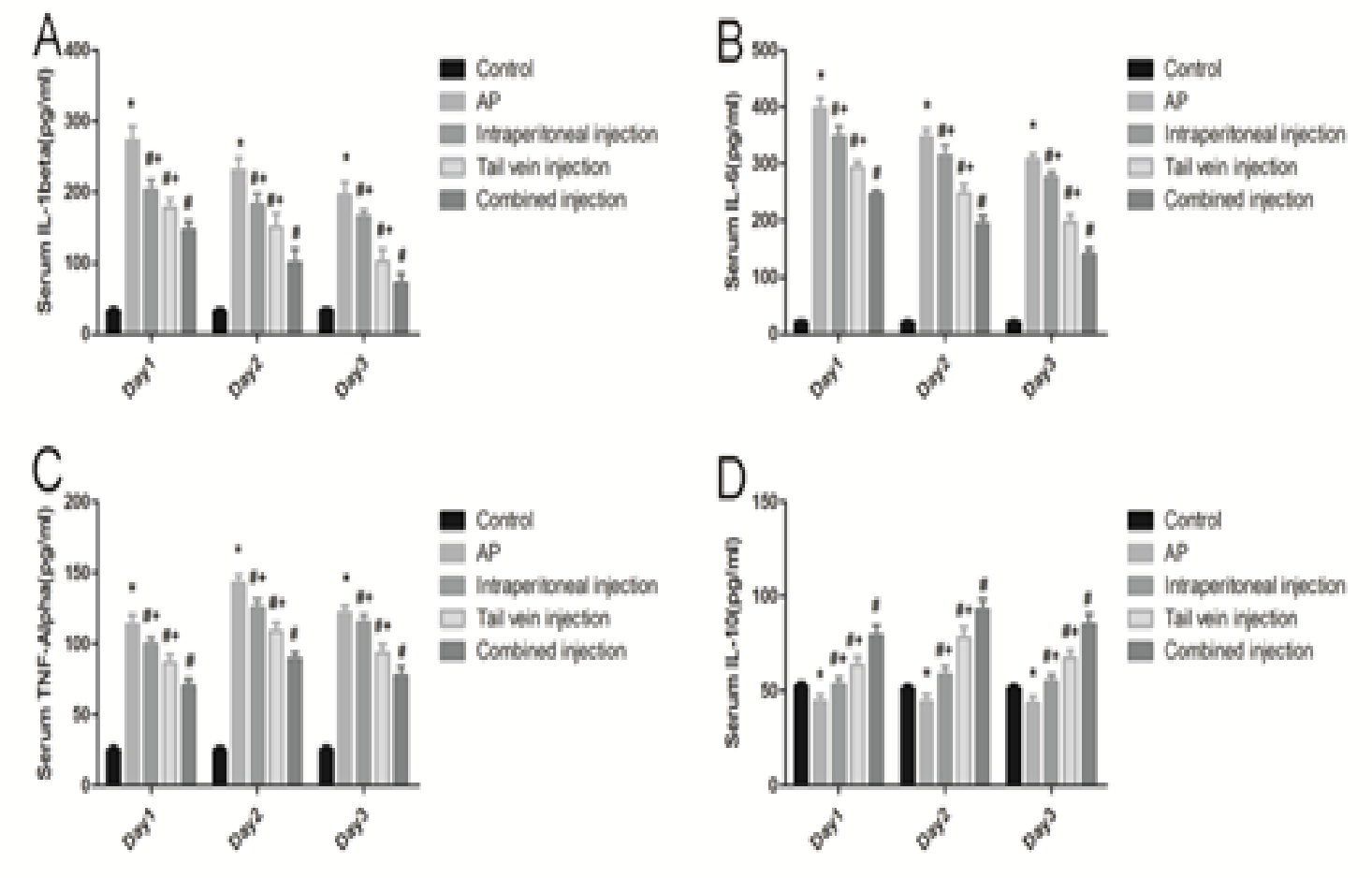

Figure 4 Inflammatory cytokines after infusion of bmMSCs. IL-I $\beta$ (A), IL-6 (B), TNF- $\alpha$ (C), and IL-I0 (D) serum levels after bmMSC infusion by enzyme-linked immunosorbent assay. $* \mathrm{P}$ means $\mathrm{P}<0.05$ when compared with Control group, \#P means $\mathrm{P}<0.05$ when compared with $\mathrm{SAP}$ group. $+\mathrm{P}$ means $\mathrm{P}<0.05$ when compared with SAP+combined injection group

Day1
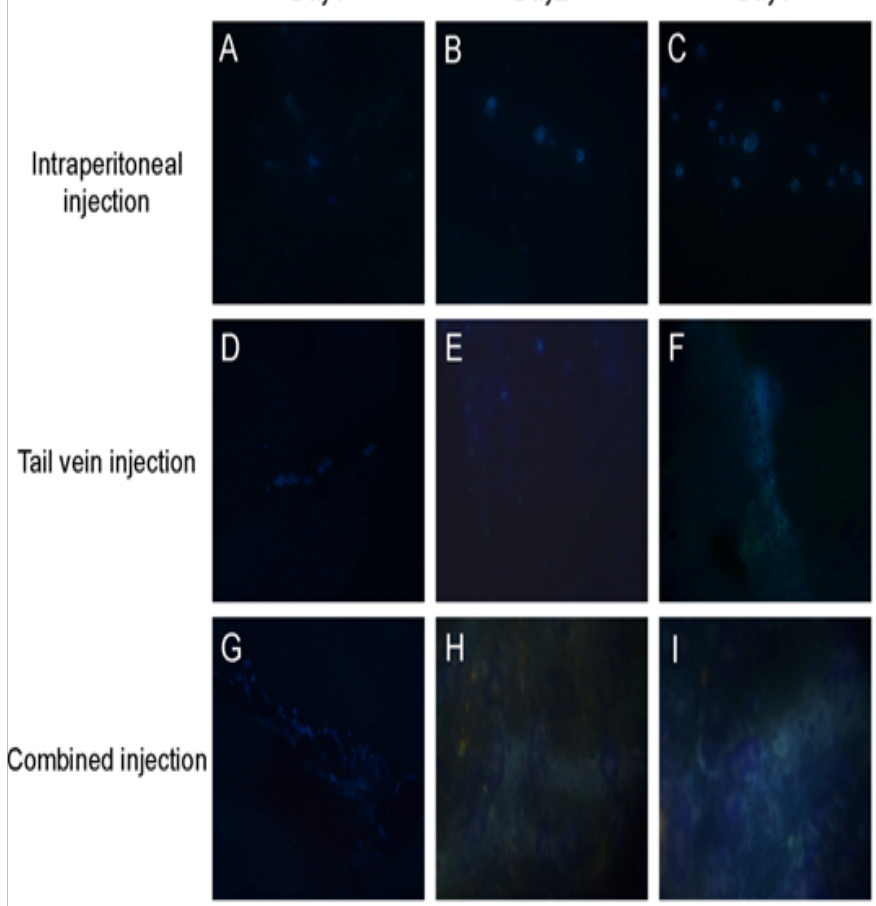

Figure 5 Tracking of infused hcMSCs. Pancreas sections of DAPI labeled hcMSCs at $1 \times 106$, injected into rats with SAP through intraperitoneal (A-C) tail vein(D-F) and tail vein + intraperitoneal (G-I) In vivo under fluorescence microscopy on days I, 2 and $3(\times 200)$

\section{Discussion}

AP is acute abdominal diseases and characterized elevated serum amylase and lipase, acinar cells necrosis, pancreatic edema, and inflammatory cells infiltration. Some patients with AP may develop SAP with lots of complications and high mortality, as yet has no satisfactory therapy. During the course of pancreatitis multiple inflammatory cytokines are reported involved in the pathogenesis of SAP including anti-inflammatory cytokines (IL-10) and proinflammatory cytokines (IL-1, IL-6, TNF- $\alpha$ ). ${ }^{12,13}$ Mesenchymal stromal cells (MSCs) as a kind of stem cells can differentiat into various adult cells and tissue and can inhibit the inflammatory response. ${ }^{14}$ To date, accumulated evidence from preclinical and linical studies has confirmed that MSCs conferred biological and functional protections in diabetes, myocardium infarction, brain and spinal cord injury etc. ${ }^{15}$ In re-sent years some experiment have reported the protective roles of MSCs in $\mathrm{AP}^{16-20}$ MSCs infusion through tail vein can protect the structural integration of acinar cells, promoted pancreatic angiogenesis, significantly inhibited inflammation and attenuate acinar cell apoptosis. Sun XC found that intraperitoneal injection of MSCs exerted protective effects on pancreas and small intestine injury. ${ }^{21}$ The goal of this study was to explore the appropriate way for the stem cells infuse In vivo. In the previous study of our group, bmMSCs were transplanted through tail vein, intraperitoneal and tail vein + intraperitoneal respectivelyl $3 \mathrm{~h}$ after SAP, and then the number of bmMSCs migrated into pancreatic and the therapeutic effect of bm-MSCs was compared. Sodium taurocholate bile duct retrograde injection was employed to induce acute pancreatitis. This animal model was feasible and easy to establish. The pancreatic enzyme detection and pathological examination reveal this animal 
model was stable. The pathological showed pancreatic edema, pancreatic parenchymal bleeding, massive inflammatory cells infiltration, and acinar cell necrosis. The bone marrow derived MSCs were injected into SAP rats via the tail vein, intraperitoneal or tail vein+intraperitoneal respectively. Some studies showed that MSCs could migrate into pancreas during early phase in AP. ${ }^{16,17}$ Consistent with recent studies, bmMSCs presented in pancreas on days 1,2 and 3 when we injected bmMSCs through tail vein or through tail vein + intraperitoneal. However we observed that bmMSCs were nearly could not be presented in pancreas on days 1,2 and 3 when we transplanted bmMSCs through intraperitoneal. It was demonstrated that bmMSCs could not migrate into pancreatic when they infused into intraperitoneal. In this study, we also confirmed that bmMSCs infusion significantly decreased serum amylase and lipase, relieved pancreatic lesions (edema, necrosis and inflammatory cell infiltration) caused by injection of sodium taurocholate. ${ }^{16,18}$ And we observed the better recovery of damaged pancreas when we transplanted bmMSCs through the tail vein + intraperitoneal compared with through tail vein or intraperitoneal alone. Consistently, inflammatory cytokines, including IL-1 $\beta$, IL- 6 and TNF- $\alpha$ of rats in the bmMSCs infusion groups were lower than the SAP group, whereas IL-10 (anti-inflammatory mediator) levels were higher in the bmMSCs infusion groups compared with SAP group. These results indicated that bmMSCs could regulate immune response by promoting the expression of anti-inflammatory mediators and reducing the expression of inflammatory factors in SAP rat modles. In addition, transplant bmMSC through tail vein combine with intraperitoneal could better inhibit inflammation than transplant bmMSC through tail vein or intraperitoneal alone. Previous studies have confirm that MSCs can repair the injured pancreas through migrate into the injured pancreas and differentiate into pancreatic cells. Interestingly, in this study the bmMSCs excerted the protective role in SAP althrough there was not enough bmMSCs homed and positioned in pancreas in SAP + bmMSCs (intraperitoneal) group. In recent years, MSCs are found to secret some cytokines such as interleukin 10, IL-1Reg, TGF- $\beta$, HGF, vascular endothelial growth factor and insulin-like growth factor IGF-1 in a paracrine dependent manner to exert the anti-inflammatory effect. ${ }^{22,23}$ It was indicated that the bmMSCs' protective role in SAP+bmMSCs (intraperitoneal) groups was through a paracrine effect, without homing to pancreas. According to our results, the bmMSC infused through tail vein and intraperitoneal interplay to amplify the protective role in SAP.

In conclusion, our results demonstrated that transplanted bmMSCs can decrease serum amylase and lipase, alleviate pancreatic lesions (edema, inflammatory cell infiltration and necrosis) caused by injection of sodium taurocholate in a rat model of SAP. According to the results of this study, transplanted bmMSCs through tail vein+intraperitoneal could be considered the better method of choice for cell-based treatment of SAP. Of course, some problems have not yet been overcome in this study. The future research should be focused on why bmMSCs can play a better role when they were transplanted through tail vein+intraperitoneal, how to lengthen cell survival time and improve the safety of bmMSCs transplantation.

\section{Acknowledgments}

This study was supported by a National Natural Science Foundation of China 81171466.

\section{Conflict of interest}

The authors declare that there is no conflict of interest.

\section{References}

1. Frossard JL, Steer ML, Pastor CM. Acute pancreatitis. Lancet. 2008;371(9607):143-152.

2. Chan YC, Leung PS. Acute pancreatitis: animal models and recent advances in basic research. Pancreas. 2007;34(1):1-14

3. McKay CJ, Imrie CW. The continuing challenge of early mortality in acute pancreatitis. Br J Surg. 2004;91(10):1243-1244.

4. Doyle KL, Kazda A, Hort Y, et al. Differentiation of adult mouse olfactory precursor cells into hair cells In vitro. Stem Cells. 2007;25(3):621-627.

5. Karaoz E, Okcu A, Ünal ZS, et al. Adipose tissue-derived mesenchymal stromal cells efficiently differentiate into insulin-producing cells in pancreatic islet microenvironment both In vitro and In vivo. Cytotherapy. 2013;15(5):557-570.

6. Jung KH, Song SU, Yi T, et al. Human bone marrow-derived clonal mesenchymal stem cells inhibit inflammation and reduce acute pancreatitisin rats. Gastroenterology. 2011;140(3):998-1008.

7. Tu XH, Song JX, Xue XJ, et al. Role of bone marrow-derived mesenchymal stem cells in a rat model of severe acute pancreatitis. World $J$ Gastroen-terol. 2012;18(18):2270-2279.

8. Chen Z, Lu F, Fang H, et al. Effect of mesenchymal stem cells on renal injury in rats with severe acute pancreatitis. Exp Biol. Med (Maywood). 2013;238(6):687-695.

9. Liu N, Tian J, Cheng J, et al. Effect of erythropoietin on the migration of bone marrow-de-rived mesenchymal stem cells to the acute kidney injury microenvironment. Exp Cell Res. 2013;319(13):2019-2027.

10. Wang L, Tu XH, Zhao P, et al. Protective effect of transplanted bone marrow-derived mesenchymal stem cells on pancreatitis-associated lung injury in rats. Mol Med Rep. 2012;6(2):287-292.

11. Wu Q, Wang F, Hou Y, et al. The effect of allogenetic bone marrow-derived mesenchymal stem cell transplantation on lung aquaporin-1 and -5 in a rat model of severe acute pancreatitis. Hepato gastroenterology. 2012;59:965-976.

12. De Waal Malefyt R, Abrams J, Bennett B, et al. Interleukin 10(IL-10) inhibits cytokine synthesis by human monocytes: an autoregulatory role of IL-10 produced by monocytes. J Exp Med. 1991;174(5):1209-1220.

13. Zyromski N, Murr MM. Evolving concepts in the pathophysiology of acute pancreatitis. Surgery. 2003;133(3):235-237.

14. Karp JM, Leng Teo GS. Mesenchymal stem cell homing: The devil is in the details. Cell Stem Cell. 2009;4(3):206-216.

15. Phinney DG, Prockop DJ. Concise review: Mesenchymal stem/multipotent stromal cells: The state of transdifferentiation and modes of tissue repair--current views. Stem Cells. 2007;25(11):2896-2902.

16. Jung KH, Song SU, Yi T, et al. Human bone marrow-derived clonal mesenchymal stem cells inhibit inflammation and reduce acute pancreatitis in rats. Gastroen-terology. 2011;140(3):998-1008.

17. Gong J, Meng HB, Hua J, et al. The sdf-1/cxcr4 axis regulates migration of transplanted bone marrow mesenchymal stem cells towards the pancreas in rats with acute pancreatitis. Mol Med Rep. 2014;9(5):1575-1582.

18. Yang B, Bai B, Liu CX, et al. Effect of umbilical cord mesenchymal stem cells on treatment of severe acute pancreatitis in rats. Cytotherapy. 2013;15(2):154-162.

19. Tu XH, Song JX, Xue XJ, et al. Role of bone marrow-derived mesenchymal stem cells in a rat model of severe acute pancreatitis. World $J$ Gastroen-terol. 2002;18(18):2270-2279. 
20. Liu N, Tian J, Cheng J, et al. Effect of erythropoietin on the migration of bone marrow-de-rived mesenchymal stem cells to the acute kidney injury microenvironment. Exp Cell Res. 2013;319(13):2019-2027.

21. Sun XC, Wu JS, Wu JM, et al. Effects of intraperitoneal injection of marrow mesenchymal stem cells on intestinal barrierin acutepancreatitis. Zhonghua Yi Xue Za Zhi. 2013;293(12):951-955.
22. Pittenger M. Sleuthing the source of regeneration by MSCs. Cell Stem Cell. 2009;5(1):8-10.

23. Lee RH, Pulin AA, Seo MJ, et al. Intravenous hMSCs improve myocardial infarction in mice because cells embolized in lung are activated to secrete the anti-inflammatory protein TSG-6. Cell Stem Cell. 2009;5(1):54-63. 